\title{
A family of equivalent norms for Lebesgue spaces
}

\author{
Alberto Fiorenza and Pankaj Jain
}

\begin{abstract}
If $\psi:[0, \ell] \rightarrow[0, \infty[$ is absolutely continuous, nondecreasing, and such that $\psi(\ell)>\psi(0), \psi(t)>0$ for $t>0$, then for $f \in L^{1}(0, \ell)$, we have$$
\|f\|_{1, \psi,(0, \ell)}:=\int_{0}^{\ell} \frac{\psi^{\prime}(t)}{\psi(t)^{2}} \int_{0}^{t} f^{*}(s) \psi(s) d s d t \approx \int_{0}^{\ell}|f(x)| d x=:\|f\|_{L^{1}(0, \ell)},
$$

where the constant in $\gtrsim$ depends on $\psi$ and $\ell$. Here by $f^{*}$ we denote the decreasing rearrangement of $f$. When applied with $f$ replaced by $|f|^{p}$, $1<p<\infty$, there exist functions $\psi$ so that the inequality $\left\||f|^{p}\right\|_{1, \psi,(0, \ell)} \leq$ $\left\||f|^{p}\right\|_{L^{1}(0, \ell)}$ is not rougher than the classical one-dimensional integral Hardy inequality over bounded intervals $(0, \ell)$. We make an analysis on the validity of $(*)$ under much weaker assumptions on the regularity of $\psi$, and we get a version of Hardy's inequality which generalizes and/or improves existing results.
\end{abstract}

Mathematics Subject Classification. 26D10, 26D15, 46E30.

Keywords. Integral inequalities, Lebesgue spaces, Weighted Lebesgue spaces, Banach function space norms, GГ spaces, Absolutely continuous functions, Monotone functions.

1. Introduction. As a by-product of a characterization of an interpolation space between grand and small Lebesgue spaces, in [7, Theorems 6.2 and 6.4] (see also [1] for recent developments), it has been shown, in particular, that for $1<p<\infty$,

$$
\left[\int_{0}^{1}\left(\int_{0}^{t}(1-\log s)^{-1} f^{*}(s)^{p} d s\right) \frac{d t}{t}\right]^{\frac{1}{p}} \approx\left(\int_{0}^{1}|f(x)|^{p} d x\right)^{\frac{1}{p}}
$$

where $f^{*}$ denotes the decreasing rearrangement of $f$. The goal of this note is to give a direct proof of a generalized version of this equivalence for functions 
defined on intervals $(0, \ell)$, true for $1 \leq p<\infty$, and with the constants of the equivalence independent of $p$. The generalization consists of the replacement of $(1-\log s)^{-1}$ by a function $\psi(s)$, and, with suitable choices of $\psi$, allows to get an inequality of the type

$$
\|f\|_{p, \psi,(0,1)} \leqslant\|f\|_{L^{p}(0,1)}
$$

not rougher than the classical one-dimensional integral Hardy inequality (see e.g. [11, Theorem 330], [19, (3.6) p. 23], or [20, Theorem 6 p. 726])

$$
\int_{0}^{1}\left(\frac{1}{x} \int_{0}^{x}|f(t)| d t\right)^{p} d x \leqslant\left(\frac{p}{p-1}\right)^{p} \int_{0}^{1}|f(x)|^{p} d x
$$

in the sense that (see Proposition 3.1 for the precise statement) there exists a family of functions $\left\{\psi_{q}\right\}_{q>0}$ such that for every $f$ and $q$ sufficiently large,

$$
\left(\frac{p-1}{p}\right)^{p} \int_{0}^{1}\left(\frac{1}{x} \int_{0}^{x} f(t) d t\right)^{p} d x \leqslant\|f\|_{p, \psi_{q},(0,1)}^{p} \leqslant \int_{0}^{1} f(x)^{p} d x .
$$

In spite of the fact that the main result (see Theorem 2.1) consists of a kind of inequality quite familiar in the literature (we quote, for instance, [21, Example 6.17 (ii) p. 330]; see also the final section), we believe that it can inspire new equivalent norms for several concrete examples of Banach function spaces, whose norm is built from that one of the Lebesgue spaces.

2. The main result. Let $0<\ell<\infty$. Denote by $\mathcal{M}$ the set of the a.e. finite, Lebesgue measurable functions defined in $(0, \ell)$. For every function $f \in \mathcal{M}$, its distribution function $\lambda_{f}:(0, \infty) \rightarrow \mathbb{R}_{+}$is defined by

$$
\lambda_{f}(s):=|\{x \in(0, \ell):|f(x)|>s\}|, \quad s>0,
$$

where by $|E|$ we mean the Lebesgue measure of $E \subset(0, \ell)$, and its decreasing rearrangement $f^{*}:(0, \ell) \rightarrow \mathbb{R}_{+}$is defined by

$$
f^{*}(t):=\inf \left\{s>0: \lambda_{f}(s) \leq t\right\}, \quad t \in(0, \ell) .
$$

Three standard properties of this operator are needed in the sequel (see e.g. [3, Propositions 1.7 and 1.8 Ch. 2]):

$$
\begin{aligned}
& f^{*} \text { is nonincreasing in }(0, \ell), \\
& \left(|f|^{p}\right)^{*}=\left(f^{*}\right)^{p} \quad \forall 1 \leqslant p<\infty, \quad f \in \mathcal{M}, \\
& \int_{0}^{\ell} f^{*}(t)^{p} d t=\int_{0}^{\ell}|f(x)|^{p} d x, \quad 1 \leqslant p<\infty .
\end{aligned}
$$

We are going to prove the following

Theorem 2.1. If $\psi:[0, \ell] \rightarrow[0, \infty[$ is absolutely continuous, nondecreasing, and such that

$$
\psi(\ell)>\psi(0), \quad \psi(t)>0 \text { for } t>0
$$


then, for every $f \in L^{1}(0, \ell)$,

$$
\|f\|_{1, \psi,(0, \ell)}:=\int_{0}^{\ell} \frac{\psi^{\prime}(t)}{\psi(t)^{2}} \int_{0}^{t} f^{*}(s) \psi(s) d s d t \approx \int_{0}^{\ell}|f(x)| d x=:\|f\|_{L^{1}(0, \ell)},
$$

where the constant in $\gtrsim$ depends on $\psi$ and $\ell$.

Proof. At first we observe that, since $\psi \geqslant 0$ is nondecreasing, using (2.4), we have

$$
0 \leqslant \frac{1}{\psi(t)} \int_{0}^{t} \psi(s) d s=\int_{0}^{t} \frac{\psi(s)}{\psi(t)} d s \leqslant \int_{0}^{t} d s=t, \quad t \text { small },
$$

and therefore

$$
\lim _{t \rightarrow 0} \frac{1}{\psi(t)} \int_{0}^{t} \psi(s) d s \rightarrow 0 .
$$

Now fix $f \in L^{1}(0, \ell)$. By (2.6), we have

$$
\begin{aligned}
\int_{0}^{\ell} \frac{\psi^{\prime}(t)}{\psi(t)^{2}} \int_{0}^{t} f^{*}(s) \psi(s) d s d t= & {\left[-\frac{1}{\psi(t)} \int_{0}^{t} f^{*}(s) \psi(s) d s\right]_{t \rightarrow 0}^{t=\ell} } \\
& -\int_{0}^{\ell}\left(-\frac{1}{\psi(t)}\right) f^{*}(t) \psi(t) d t \\
= & \int_{0}^{\ell} f^{*}(t)\left(1-\frac{\psi(t)}{\psi(\ell)}\right) d t
\end{aligned}
$$

Since $\psi$ is nonnegative, by (2.3), we immediately have that

$$
\int_{0}^{\ell} \frac{\psi^{\prime}(t)}{\psi(t)^{2}} \int_{0}^{t} f^{*}(s) \psi(s) d s d t \leqslant \int_{0}^{\ell} f^{*}(t) d t=\int_{0}^{\ell}|f(x)| d x .
$$

On the other hand, by the continuity of $\psi$ and by (2.4), we have

$$
\{\zeta \in(0, \ell): \psi(\zeta)<\psi(\ell)\} \neq \emptyset,
$$

and therefore, setting

$$
\tau_{0}:=\sup \{\zeta \in(0, \ell): \psi(\zeta)<\psi(\ell)\} \in(0, \ell],
$$

we are allowed to fix $\tau \in\left(0, \tau_{0}\right)$. We now estimate separately the integrals $\int_{0}^{\tau} f^{*}(t) d t$ and $\int_{\tau}^{\ell} f^{*}(t) d t$. 
Since $\psi$ is nondecreasing, and using the relation $\tau<\ell$, we have

$$
\begin{aligned}
\int_{0}^{\tau} f^{*}(t) d t & =\int_{0}^{\tau} f^{*}(t)\left(1-\frac{\psi(t)}{\psi(\ell)}\right) \frac{1}{1-\frac{\psi(t)}{\psi(\ell)}} d t \\
& \leqslant \frac{1}{1-\frac{\psi(\tau)}{\psi(\ell)}} \int_{0}^{\tau} f^{*}(t)\left(1-\frac{\psi(t)}{\psi(\ell)}\right) d t \\
& \leqslant \frac{1}{1-\frac{\psi(\tau)}{\psi(\ell)}} \int_{0}^{\ell} f^{*}(t)\left(1-\frac{\psi(t)}{\psi(\ell)}\right) d t .
\end{aligned}
$$

On the other hand, by (2.1), since $\psi$ is nondecreasing and $\tau<\tau_{0}$, we have $\psi(\tau)<\psi(\ell)$ and therefore

$$
\begin{aligned}
\int_{\tau}^{\ell} f^{*}(t) d t & \leqslant \int_{\tau}^{\ell} f^{*}(\tau) d t \\
& =f^{*}(\tau)(\ell-\tau) \\
& =\frac{1}{\tau} \int_{0}^{\tau} f^{*}(\tau)(\ell-\tau) d t \\
& =\frac{1}{\tau} \int_{0}^{\tau} f^{*}(\tau)\left(1-\frac{\psi(\tau)}{\psi(\ell)}\right) \frac{\ell-\tau}{1-\frac{\psi(\tau)}{\psi(\ell)}} d t \\
& \leqslant \frac{1}{\tau} \cdot \frac{\ell-\tau}{1-\frac{\psi(\tau)}{\psi(\ell)}} \int_{0}^{\ell} f^{*}(t)\left(1-\frac{\psi(t)}{\psi(\ell)}\right) d t .
\end{aligned}
$$

Joining (2.9) and (2.10), for every fixed $\tau$, we have

$$
\int_{0}^{\ell} f^{*}(t) d t \leqslant\left(\frac{1}{1-\frac{\psi(\tau)}{\psi(\ell)}}+\frac{1}{\tau} \cdot \frac{\ell-\tau}{1-\frac{\psi(\tau)}{\psi(\ell)}}\right) \int_{0}^{\ell} f^{*}(t)\left(1-\frac{\psi(t)}{\psi(\ell)}\right) d t
$$

and finally, setting

$$
C_{\psi, \ell}:=\inf _{\tau \in\left(0, \tau_{0}\right)}\left(\frac{1}{1-\frac{\psi(\tau)}{\psi(\ell)}}+\frac{1}{\tau} \cdot \frac{\ell-\tau}{1-\frac{\psi(\tau)}{\psi(\ell)}}\right)=\inf _{\tau \in\left(0, \tau_{0}\right)} \frac{\ell \psi(\ell)}{\tau(\psi(\ell)-\psi(\tau))}>0
$$

and taking into account the identities (2.3), (2.7), we get

$$
\int_{0}^{\ell}|f(x)| d x \leq C_{\psi, \ell} \int_{0}^{\ell} \frac{\psi^{\prime}(t)}{\psi(t)^{2}} \int_{0}^{t} f^{*}(s) \psi(s) d s d t
$$

and we are done. 
3. The connection with the Hardy inequality. The $p$-convexification (for details, see e.g. [28, p. 179], [13,29]) of the left hand side of (2.5)

$$
\|f\|_{p, \psi,(0, \ell)}:=\left\|f^{p}\right\|_{1, \psi,(0, \ell)}^{\frac{1}{p}}=\left(\int_{0}^{\ell} \frac{\psi^{\prime}(t)}{\psi(t)^{2}} \int_{0}^{t} f^{*}(s)^{p} \psi(s) d s d t\right)^{\frac{1}{p}},
$$

in the assumptions of Theorem 2.1 (the equality holds by (2.2)), is an equivalent norm for $L^{p}(0, \ell)$ for $1 \leq p<\infty$. On the other hand, the left hand side of the Hardy inequality (1.2) is an equivalent norm for $L^{p}(0,1)$ when $1<p<\infty$, and the degeneracy at $p=1$ tells us that such an equivalent norm is not the $p$-convexification of a norm for $L^{1}(0,1)$.

After the proof of Theorem 2.1, and taking into account Hardy's inequality, we can assert that when $1<p<\infty$, both the expressions

$$
\begin{aligned}
{[f]_{L^{p}(0,1)}^{p}:=} & \left(\frac{p-1}{p}\right)^{p} \int_{0}^{1}\left(\frac{1}{x} \int_{0}^{x} f^{*}(t) d t\right)^{p} d x \quad \text { and } \\
\|f\|_{p, \psi,(0,1)}^{p} & =\int_{0}^{1} \frac{\psi^{\prime}(t)}{\psi(t)^{2}} \int_{0}^{t} f^{*}(s)^{p} \psi(s) d s d t
\end{aligned}
$$

are norms, raised to the power $p$, for Lebesgue spaces $L^{p}(0,1)$, both of them smaller than the standard norm $\|f\|_{L^{p}(0,1)}=\int_{0}^{1}|f(x)|^{p} d x$. Note that the constant in the right hand side of the classical Hardy inequality (1.2) is optimal (see e.g. [19, Example 1 p. 20]), and therefore the inequality $[f]_{L^{p}(0,1)}^{p} \leqslant$ $\|f\|_{L^{p}(0,1)}$ would not hold for all $f$ if the constant $\left(\frac{p-1}{p}\right)^{p}$ in $[f]_{L^{p}(0,1)}^{p}$ would be replaced by a greater one.

We are going to show that there exist suitable choices for $\psi$ so that the inequality $\|f\|_{p, \psi,(0,1)} \leqslant\|f\|_{L^{p}(0,1)}$, equivalent to that one established in Theorem 2.1, is not rougher than (and therefore it cannot be deduced as a consequence of) the classical Hardy inequality.

Proposition 3.1. Let $1<p<\infty$ and $q>0$, and set $\psi_{q}(t):=(1-\log t)^{-q}$, $t \in(0,1)$, extended in $t=0$ by continuity. If $q$ is sufficiently large, there exist functions $f_{0}$ such that

$$
\left[f_{0}\right]_{L^{p}(0,1)} \leqslant\left\|f_{0}\right\|_{p, \psi_{q},(0,1)} \leqslant\left\|f_{0}\right\|_{L^{p}(0,1)} .
$$

Proof. We already observed that the right wing inequality in (3.1) holds for every $f \in L^{p}(0,1)$, therefore we need to prove the assertion only for the left wing inequality.

The optimality of the constant in the right hand side of the classical Hardy inequality (1.2) can be expressed writing

$$
\sup _{f \not \equiv 0} \frac{[f]_{L^{p}(0,1)}^{p}}{\|f\|_{L^{p}(0,1)}^{p}}=1 ;
$$


moreover, the proof of the optimality (see e.g. [19, Example 1 p. 20]) ensures that the supremum in (3.2) is not a maximum. Therefore, if we fix any function $f_{0} \in L^{p}(0,1), f_{0} \not \equiv 0$, we have

$$
\frac{\left[f_{0}\right]_{L^{p}(0,1)}^{p}}{\left\|f_{0}\right\|_{L^{p}(0,1)}^{p}}<1
$$

on the other hand, by (2.7),

$$
\begin{aligned}
\left\|f_{0}\right\|_{p, \psi_{q},(0,1)}^{p} & =\int_{0}^{1} \frac{\psi_{q}^{\prime}(t)}{\psi_{q}(t)^{2}} \int_{0}^{t} f_{0}^{*}(s)^{p} \psi_{q}(s) d s d t=\int_{0}^{1} f_{0}^{*}(t)^{p}\left(1-\frac{\psi_{q}(t)}{\psi_{q}(1)}\right) d t \\
& =\int_{0}^{1} f_{0}^{*}(t)^{p}\left(1-\psi_{q}(t)\right) d t
\end{aligned}
$$

and since $\psi_{q}(t)=(1-\log t)^{-q} \downarrow 0$ a.e. as $q \uparrow \infty$, we have $1-\psi_{q}(t) \uparrow 1$ a.e. as $q \uparrow \infty$. By the Fatou property for Lebesgue spaces,

$$
\int_{0}^{1} f_{0}^{*}(t)^{p}\left(1-\psi_{q}(t)\right) d t \uparrow\left\|f_{0}\right\|_{L^{p}(0,1)}^{p} \quad \text { as } q \rightarrow \infty,
$$

and consequently

$$
\frac{\left\|f_{0}\right\|_{p, \psi_{q},(0,1)}^{p}}{\left\|f_{0}\right\|_{L^{p}(0,1)}^{p}} \uparrow 1 \quad \text { as } q \rightarrow \infty .
$$

By (3.3), it is now clear that for $q$ large, it must be

$$
\frac{\left[f_{0}\right]_{L^{p}(0,1)}^{p}}{\left\|f_{0}\right\|_{L^{p}(0,1)}^{p}}<\frac{\left\|f_{0}\right\|_{p, \psi_{q},(0,1)}^{p}}{\left\|f_{0}\right\|_{L^{p}(0,1)}^{p}}
$$

from which the claim follows.

The degeneracy of the Hardy inequality when $p \rightarrow 1$ causes the appearance of a logarithm. A direct application of an integration by parts gives (see e.g. [11, 240 p. 169])

$$
\int_{0}^{1} \frac{1}{x} \int_{0}^{x}|f(t)| d t d x=\int_{0}^{1}|f(x)| \log \left(\frac{1}{x}\right) d x .
$$

When (3.4) is considered for decreasing functions, the equality (important because both sides are equivalent norms for the Zygmund space $L \log L$ ) may take the form (see e.g. [3, (6.7) p. 245]):

$$
\int_{0}^{1} \frac{1}{t} \int_{0}^{t} f^{*}(s) d s d t=\int_{0}^{1} f^{*}(x) \log \left(\frac{1}{x}\right) d x .
$$


It may be of interest to observe that the factor $1 / t$ in the integrand in the left hand side is exactly $\psi^{\prime}(t) / \psi^{2}(t)$ if we set

$$
\psi(t)=\frac{1}{\log \left(\frac{1}{t}\right)} .
$$

Strictly speaking, this choice is not allowed in Theorem 2.1 (even considering the extension by continuity in $t=0$ ) because in $t=1$ the function $\psi$ diverges and continuity in the closed interval $[0,1]$ is lost. However, we remark that the proof of Theorem 2.1 works as well changing $\psi(\ell)$ into $\psi(\ell-)$, which is allowed to be infinite (in such case, considering, as usual, $1 / \psi(\ell-)=0$ ).

The substitution of (3.6) into (2.5), applied with $f(x)$ replaced by $f(x) \log \left(\frac{1}{x}\right)$, gives the interesting complement to $(3.5)$

$$
\int_{0}^{1} \frac{1}{t} \int_{0}^{t}\left(f(\cdot) \log \left(\frac{1}{\cdot}\right)\right)^{*}(s) \frac{1}{\log \left(\frac{1}{s}\right)} d s d t \approx \int_{0}^{1}|f(x)| \log \left(\frac{1}{x}\right) d x
$$

when $f$ is nonincreasing, taking into account that the product of positive nonincreasing functions is still nonincreasing, (3.7) tells that equivalence holds in $(3.5)$.

\section{Remarks on the main result.}

4.1. The necessity of a monotonicity assumption on $\psi$. In Theorem 2.1 , the function $\psi$ is assumed to be nondecreasing so that in $[0, \ell]$, the existence of subintervals in which $\psi$ is constant is allowed. However, $\psi$ cannot be constant in the whole of $[0, \ell]$ because of the assumption (2.4). Note that if $\psi$ is a constant function, then the equivalence (2.5) cannot hold because the left hand side would be 0 (and therefore only the inequality $\leqslant$ remains true and trivial).

4.2. The necessity of the absolute continuity of $\psi$. The equivalence of Theorem 2.1 involves the a.e. derivative of $\psi$, which is assumed to be nondecreasing. A classical theorem by Lebesgue (see e.g. [5, Theorem 3.1 p. 176]) states that nondecreasing functions are a.e. differentiable, and therefore, more generally, functions of bounded variation (which are the differences of monotone functions) are a.e. differentiable. However, the assumption of absolute continuity of $\psi$ cannot be dropped. In fact, the Cantor ternary function (see e.g. [5, 5.5 p. 210]) is a continuous, strictly increasing function in $[0,1]$, whose derivative vanishes a.e. in $[0,1]$. It is clear that if $\psi$ would be the Cantor ternary function, the left hand side of (2.5) would be 0 and the equivalence would not hold. The absolute continuity of $\psi$ has been used in the proof while using the integration by parts (see e.g. [4, Corollary 20.20 p. 375] or [5, 7.3 p. 213]).

4.3. Extension to the case of $\psi$ of bounded variation. In spite of the necessity of the absolute continuity of $\psi$ in Theorem 2.1, a small change in (2.5) can extend the validity of the equivalence also to the case of functions $\psi$ of 
bounded variation (and therefore to a class of functions which may be possibly discontinuous). In fact, with the same proof of Theorem 2.1, we have

$$
\int_{0}^{\ell} \int_{0}^{t} f^{*}(s) \psi(s) d s d\left(-\frac{1}{\psi(t)}\right) \approx \int_{0}^{\ell}|f(x)| d x .
$$

The validity of the integration by parts formula, in this case, holds for integrals in the Stieltjes sense (see e.g. [4, Theorem 14.10 p. 227]). In order to make the machinery work, we need, however, that $\psi$ is positive and strictly increasing, so that also $-1 / \psi$ is positive and strictly increasing (which implies that it is of bounded variation).

4.4. Removing any regularity assumption on $\psi$. If $\psi$ is not assumed to be absolutely continuous, but just nondecreasing, the equivalence (in general) does not hold (as we already saw, considering the Cantor ternary function). However, the inequality $\leqslant$ in $(2.5)$ is still true if one sticks to the formulation with $\psi^{\prime}$ because $\psi$ admits the a.e. derivative as well (because of the classical theorem by Lebesgue, see e.g. [5, Theorem 3.1 p. 176]) and both the fundamental theorem of calculus, and integration by parts are true with inequality $\leqslant$ instead of equality (see e.g. [18, (1.20) p. 12]).

4.5. Relevant special cases. In the case of the functions $\psi_{q}$ in Proposition 3.1, we have $\psi_{q}(t)=(1-\log t)^{-q}, t \in(0,1), q>0$, and therefore

$$
\frac{\psi_{q}^{\prime}(t)}{\psi_{q}(t)^{2}}=\frac{q(1-\log t)^{q-1}}{t} .
$$

When $q=1$,

$$
\frac{\psi_{1}^{\prime}(t)}{\psi_{1}(t)^{2}}=\frac{1}{t}
$$

and (2.5) reduces to (1.1).

Elementary but tedious computations show that when $\psi_{q}(t)$ is multiplied by a power $t^{\lambda}, \lambda \geqslant 0$, the inequality $\leqslant$ proved in Theorem 2.1 fits into the special case of $b=1$ and $\Phi$ decreasing of a celebrated inequality proved in Bennett-Rudnik [2, Theorem 6.4] (see also [7, Theorem 2.1]). It is interesting to observe that, in this special situation, Theorem 2.1 guarantees the validity of the reverse inequality, too.

5. Comparison with some existing results. The literature about integral inequalities for real valued functions, more or less related to variants and generalizations of the Hardy inequality, is huge: it starts even before Hardy (the references in the celebrated [11] are a proof), it grew in the sixties and in the seventies of the last century, and it continues to grow until now, see e.g. $[14,15,22]$. The development has been in several directions, among which the framework in Banach spaces other than Lebesgue spaces (see e.g. the treatment [19, Chapter 11], [8], and [13]). Any comparison with existing results has to be incomplete, however, we will try to mention some references at our knowledge. It will be of help to record some features of (2.5) in Theorem 2.1: 
the equivalence is between both terms; (when applied to $f^{p}$ :) the validity is for $p \geq 1$, hence the case $p=1$ is included; the left hand side involves nonincreasing functions; the function $\psi$ is not necessarily concave and does not necessarily satisfy $\psi(0)=0$.

5.1. The first weighted modification of Hardy's inequality. A classical weighted Hardy inequality (see e.g. [19, (3.6) p. 23], [21, (0.4) p. 2], see also $[6,(4),(5)$ p. 494]) states that

$$
\int_{0}^{\infty}\left(\frac{1}{x} \int_{0}^{x}|f(t)| d t\right)^{p} x^{\alpha} d x \leqslant\left(\frac{p}{p-\alpha-1}\right)^{p} \int_{0}^{\infty}|f(x)|^{p} x^{\alpha} d x
$$

is valid with $p>1$ and $\alpha<p-1$. Let us consider the special case of $\alpha<0$ and $g(x):=f(x) x^{\frac{\alpha}{p}}$ nonincreasing (which means that the integrand on the right hand side is nonincreasing) and supported in an interval $[0, \ell]$. In terms of $g$, (5.1) gives the validity of the following inequality:

$$
\int_{0}^{\ell}\left(\frac{1}{x} \int_{0}^{x} g^{*}(t) t^{-\frac{\alpha}{p}} d t\right)^{p} x^{\alpha} d x \leqslant\left(\frac{p}{p-\alpha-1}\right)^{p} \int_{0}^{\ell}|g(x)|^{p} d x .
$$

An application of Hölder's inequality to the left hand side makes the inequality, a priori, either false, or an improvement. Well, using (2.2), we know that the inequality

$$
\int_{0}^{\ell} \frac{1}{x} \int_{0}^{x} g^{*}(t)^{p} t^{-\alpha} d t x^{\alpha} d x \leqslant\left(\frac{p}{p-\alpha-1}\right)^{p} \int_{0}^{\ell}|g(x)|^{p} d x,
$$

which as $p \rightarrow 1$ reads

$$
\int_{0}^{\ell} \frac{1}{x} \int_{0}^{x} g^{*}(t) t^{-\alpha} d t x^{\alpha} d x \leqslant \frac{1}{-\alpha} \int_{0}^{\ell}|g(x)| d x=\frac{1}{|\alpha|} \int_{0}^{\ell}|g(x)| d x,
$$

is true, setting $\psi(t)=t^{-\alpha}$ in (2.8) (note that it is already independently known, see $[11,330$ p. 245 , case $p=1]$, $[12,33])$. The same considerations hold starting from the inequality (see $[32,(1.2)$ p. 1])

$$
\int_{0}^{\infty}\left(\frac{1}{x} \int_{0}^{x} g(y) d y\right)^{p} \frac{d x}{x} \leq \int_{0}^{\infty} g(x)^{p} \frac{d x}{x} .
$$

5.2. The two-weighted Hardy operator. An important, nowadays classical, result (see the Talenti-Tomaselli-Muckenhoupt theorem [19, Theorem 4 p. 38]) contains a necessary and sufficient condition for the validity of the following two-weighted ( $u, v$ nonnegative and locally integrable) inequality

$$
\left(\int_{0}^{\ell}\left(\int_{0}^{x}|f(t)| d t\right)^{p} u(x) d x\right)^{\frac{1}{p}} \leq C\left(\int_{0}^{\ell}|f(x)|^{p} v(x) d x\right)^{\frac{1}{p}} .
$$


Namely, if $1 \leqslant p<\infty$ (note that $p=1$ is allowed), (5.2) holds if and only if

$$
\sup _{r \in(0, \ell)}\left(\int_{r}^{\ell} u(x) d x\right)^{\frac{1}{p}}\left(v(x)^{1-p^{\prime}} d x\right)^{1 / p^{\prime}}<\infty \quad\left(p^{\prime}=p /(p-1)\right) .
$$

We remark that one inequality of the equivalence (2.5) fits completely in the case $p=1$ of (5.2). However, we deal with nonincreasing functions $f$ and with weights of special form (given the nondecreasing function $\psi$, one has to put in (2.5), $\psi(t)=v(t)^{-1}, \psi^{\prime}(t) / \psi^{2}(t)=u(t)$, and then setting $g(x)=f(x) v(x)$, one gets back (5.2)), and this allows, for us, to get an equivalence. Note that it would make no sense to check, for our case, condition (5.3) because when $f$ is nonincreasing and $\psi$ nondecreasing, the left hand side of (2.5), by a celebrated Hardy inequality for rearrangements, is smaller than the left hand side of (5.2).

We omit here to quote the literature nicely described in [30, Chapter 1] about two-weighted inequalities, and in [19, Chapter 10] and [16] about the weighted Hardy inequality for monotone functions: even if several results contain inequalities very close to our (2.5), sometimes allowing $p=1$ and/or nonincreasing functions, essentially all of them are not equivalences.

5.3. The average operators. In [19, p. 129], a description of a series of papers ([24-27]) appears (see also [23, Theorem 2] and [17]), all of them being devoted to the boundedness of operators of the type

$$
f \rightarrow \frac{1}{W(x)} \int_{0}^{x} f(t) w(t) d t
$$

in various Banach spaces, where $W(x)=\int_{0}^{x} w(t) d t$. They generalize, setting $w(t)=1$, the Hardy operator

$$
f \rightarrow \frac{1}{t} \int_{0}^{t} f(s) d s
$$

We remark that also the operator in (2.5)

$$
f \rightarrow \frac{\psi^{\prime}(t)}{\psi(t)^{2}} \int_{0}^{t} f^{*}(s) \psi(s) d s
$$

may lead to the same operator using (3.6): the factor of the integral is again $1 / t$, but we "pay the price" of the factor $\psi$ in the integrand, which is nondecreasing and also multiplied by a nonincreasing function. The counterpart is that we "gain" the estimate of the operator in $L^{1}$.

5.4. More weighted variants of Hardy's inequality. In [31], quite technical generalizations of Hardy-type inequalities appear, involving weights $\left\{r_{n}\right\}, z, w$. When considering the basic case $w \equiv r_{1} \equiv 1$, setting $z^{m-1}=\psi$, we have $z^{\prime} / z^{m}=\psi^{\prime} / \psi^{2}$ and with some tedious computations, it is possible to check that the main inequality in [31] coincides with the $\leqslant$ in $(2.5)$. However, due to the general framework of [31], the authors need to assume $z^{\prime}>0$ and 
$z(0+)>0$, also to have the possibility to deal with functions $f$ not necessarily monotone. Similarly, other inequalities where more weights are involved, again only in one direction, appear in [9].

5.5. A special Generalized Gamma space with double weights. The quasinorm of the Generalized Gamma space with double weights $G \Gamma\left(p, m ; w_{1}, w_{2}\right)$ (see $[7,10]$ for details and comments on this notion) is given by

$$
\rho(f)=\left[\int_{0}^{1} w_{1}(t)\left(\int_{0}^{t} f^{*}(s)^{p} w_{2}(s) d s\right)^{\frac{m}{p}} d t\right]^{\frac{1}{m}} .
$$

It is known that Generalized Gamma spaces give back, as special cases, several classical Banach spaces.

In the assumptions of Theorem 2.1, we can add one more relevant special case, namely,

$$
G \Gamma\left(1,1 ; \frac{\psi^{\prime}}{\psi^{2}}, \psi\right)=L^{1}
$$

Acknowledgement. The second author acknowledges the MATRICS Research Grant No. MTR/2017/000126 of SERB, Department of Science and Technology, India.

Funding Open access funding provided by Università degli Studi di Napoli Federico II within the CRUI-CARE Agreement.

Open Access. This article is licensed under a Creative Commons Attribution 4.0 International License, which permits use, sharing, adaptation, distribution and reproduction in any medium or format, as long as you give appropriate credit to the original author(s) and the source, provide a link to the Creative Commons licence, and indicate if changes were made. The images or other third party material in this article are included in the article's Creative Commons licence, unless indicated otherwise in a credit line to the material. If material is not included in the article's Creative Commons licence and your intended use is not permitted by statutory regulation or exceeds the permitted use, you will need to obtain permission directly from the copyright holder. To view a copy of this licence, visit http://creativecommons.org/ licenses/by/4.0/.

Publisher's Note Springer Nature remains neutral with regard to jurisdictional claims in published maps and institutional affiliations.

\section{References}

[1] Ahmed, I., Fiorenza, A., Formica, M.R., Gogatishvili, A., Rakotoson, J.M.: Some new results related to Lorentz GГ-spaces and interpolation. J. Math. Anal. Appl. 483, $123623(2020)$

[2] Bennett, C., Rudnik, K.: On Lorentz-Zygmund spaces. Dissertationes Math. (Rozrrawy Mat.) 175 (1980) 
[3] Bennett, C., Sharpley, R.: Interpolation of Operators. Pure and Applied Mathematics, vol. 129. Academic Press Inc, Boston, MA (1988)

[4] Carothers, N.L.: Real Analysis. Cambridge University Press, Cambridge (2000)

[5] DiBenedetto, E.: Real Analysis. Birkhäuser Advanced Texts. Birkhäuser, Boston (2002)

[6] Fabricant, A., Kutev, N., Rangelov, T.: Hardy-type inequalities with weights. Serdica Math. J. 41, 493-512 (2015)

[7] Fiorenza, A., Formica, M.R., Gogatishvili, A., Kopaliani, T., Rakotoson, J.M.: Characterization of interpolation between grand, small or classical Lebesgue spaces. Nonlinear Anal. 177, 422-453 (2018)

[8] Fiorenza, A., Gupta, B., Jain, P.: The maximal theorem for weighted grand Lebesgue spaces. Stud. Math. 188(2), 123-133 (2008)

[9] Fiorenza, A., Gupta, B., Jain, P.: Mixed norm type Hardy inequalities. Can. Math. Bull. 54(4), 630-644 (2011)

[10] Fiorenza, A., Rakotoson, J.M.: Some estimates in $\operatorname{G} \Gamma(p, m, w)$ spaces. J. Math. Anal. Appl. 340, 793-805 (2008)

[11] Hardy, G.H., Littlewood, J.E., Polya, G.: Inequalities. Cambridge University Press, Cambridge (1952)

[12] Imoru, C.O.: On some integral inequalities related to Hardy's. Can. Math. Bull. 20(3)(1), 307-312 (1977)

[13] Jain, P., Gupta, B., Verma, D.: Mean inequalities in certain Banach function spaces. J. Math. Anal. Appl. 334(1), 358-367 (2007)

[14] Jain, P., Kanjilal, S., Persson, L.-E.: Hardy-type inequalities over balls in $\mathbb{R}^{N}$ for some bilinear and iterated operators. J. Inequal. Spec. Funct. 10(2), 35-48 (2019)

[15] Jain, P., Kanjilal, S., Stepanov, V.-D., Ushakova, E.P.: Bilinear Hardy-Steklov operators. Math. Notes 104(5-6), 823-832 (2018)

[16] Jain, P., Singh, M., Singh, A.P.: Weighted norm inequalities for Hardy type operators on monotone functions. In: Function Spaces in Analysis. Contemporary Mathematics, vol. 645, pp. 145-160. Amer. Math. Soc, Providence, RI (2015)

[17] Jain, P., Singh, M., Singh, A.P.: Hardy-type integral inequalities for quasimonotone functions. Georgian Math. J. 24(4), 523-533 (2017)

[18] Klebaner, F.C.: Introduction to Stochastic Calculus with Applications, 3rd edn. Imperial College Press, London (2012)

[19] Kufner, A., Maligranda, L., Persson, L.E.: The Hardy inequality. About its History and Some Related Results. Vydavatelský Servis, Pilsen (2007)

[20] Kufner, A., Maligranda, L., Persson, L.E.: The prehistory of the Hardy inequality. Amer. Math. Mon. 113(8), 715-732 (2006)

[21] Kufner, A., Persson, L.E., Samko, N.: Weighted Inequalities of Hardy Type, 2nd edn. World Scientific Publishing Co. Pte. Ltd, Hackensack, NJ (2017)

[22] Kutev, N., Rangelov, T.: Hardy inequalities with double singular weights. arXiv:2001.07368v2 (2020) 
[23] Levinson, N.: Generalizations of an inequality of Hardy. Duke Math. J. 31(3), 389-394 (1964)

[24] Maligranda, L.: Generalized Hardy inequalities in rearrangement invariant spaces. J. Math. Pures Appl. (9) 59(4), 405-415 (1980)

[25] Maligranda, L.: A generalization of the Shimogaki theorem. Stud. Math. 71(1), 69-83 (1981/1982)

[26] Maligranda, L.: On Hardy's inequality in weighted rearrangement invariant spaces and applications. I, II. Proc. Amer. Math. Soc. 88(1), 67-74, 75-80 (1983)

[27] Maligranda, L.: Indices and interpolation. Dissertationes Math. (Rozprawy Mat.) 234 (1985)

[28] Maligranda, L.: Orlicz spaces and interpolation. Seminários de Matemática [Seminars in Mathematics], vol. 5, Universidade Estadual de Campinas, Departamento de Matemática, Campinas (1989)

[29] Maligranda, L., Persson, L.E.: Generalized duality of some Banach function spaces. Indag. Math. 52, 323-338 (1989)

[30] Opic, B., Kufner, A.: Hardy-Type Inequalities. Longman, Harlow (1990)

[31] Pachpatte, B.G., Love, E.R.: On some new inequalities related to Hardy's integral inequality. J. Math. Anal. Appl. 149, 17-25 (1990)

[32] Persson, L.E., Samko, N.: What should have happened if Hardy had discovered this? J. Ineq. Appl. 2012, 29 (2012)

[33] Tian, J., Zhou, Y.-X.: Refinements of Hardy-type inequalities. Abstr. Appl. Anal. ArticleID 727923 (2013)

\section{Alberto Fiorenza}

Dipartimento di Architettura

Università di Napoli Federico II

Via Monteoliveto, 3

80134 Naples

Italy

and

Istituto per le Applicazioni del Calcolo "Mauro Picone", sezione di Napoli Consiglio Nazionale delle Ricerche

Via Pietro Castellino, 111

80131 Naples

Italy

e-mail: fiorenza@unina.it

PANKAJ JAIN

Department of Mathematics

South Asian University

Akbar Bhawan, Chanakya Puri

New Delhi 110021

India

e-mail: pankaj.jain@sau.ac.in 
Received: 17 July 2020

Revised: 26 August 2020

Accepted: 16 September 2020. 